\title{
Per 200 High Powered Fields
}

National Cancer Institute

\section{Source}

National Cancer Institute. Per 200 High Powered Fields. NCI Thesaurus. Code 132472.

A unit of measurement of the number of entities per unit of area equal to 200 high

powered fields. 